\title{
Orientación vocacional a distancia: perspectivas de los estudiantes de educación superior
}

\author{
Juan Manuel Álvarez, Universidad de Guanajuato, Guanajuato, México
}

Resumen: Existen diferentes tipos de orientación; este proyecto se dirige a la argumentación de la creación de una página web de orientación vocacional, que tratará de apoyar a los alumnos que quieren ingresar a una formación superior. Se les guiará con base en sus intereses, habilidades, conocimientos, y tomando en cuenta las posibilidades que el entorno les ofrece. Todo esto será un servicio creado en uso de las nuevas tecnologías a través de los medios de comunicación vía internet, enriquecida con especialistas en orientación, investigadores en educación, licenciados en diseño grafico, ingeniero en sistemas computaciones y uno o más especialistas por cada una de las carreras que ofrece la Universidad de Guanajauto, esta unión será de gran ayuda para todas aquellas personas que requieran de un guía para la toma de decisión en una carrera o profesión. La finalidad de la orientación vocacional es asistir a las personas de cualquier edad y en cualquier momento de su vida académica, e integrar opiniones educativas de formación y ejercer sus preferencias; es necesaria una ayuda para despejar las dudas de su formación profesional y posteriormente dirigirse al ámbito laboral, brindando herramientas de orientación vocacional como también profesional. Esta es una decisión muy complicada para los estudiantes, y en ella se verá reflejado gran parte de su futuro individual y colectivo. Esta plataforma pretende ser de gran utilidad en México y en otros países de habla hispana, que confrontan un cambio en la formación educativa básica y superior de su población, mismo que es producto de la acelerada transformación tecnológica.

Palabras Clave: Orientación Vocacional, Página Web, Carreras Profesionales, Guía, Habilidades, Gustos, Intereses y Tecnologías Incorporadas a la Comunicación (TIC's)

Abstract: There are different types of guidance; this project aims to vocational guidance, which aims to support students who want to enter a higher education. It will guide them based on their interests, skills, knowledge, and taking into account the possibilities that the environment offers. This is a service created in use of new technologies through the media via the Internet. The purpose of vocational guidance is to assist people of any age and at any time during their academic life, and integrate training and educational views to exercise their preferences, help is needed to clarify the doubts of their training and then go to the workplace. This is a very complicated decision for students, and it will be reflected much of their individual and collective future. This platform aims to be of great use in Mexico and other Spanish-speaking countries in developing countries, facing a change in the basic education and higher education of its population, it is the product of the rapid technological transformation.

Keywords: Vocational Guidance, Website, Professional Careers, Guidance, Skills, Preferences, Interests and Technologies in the Communication (ICT)

Revista Internacional de Educaci ón y Aprendizaje

Volumen 1, 2013, http://sobrelaeducacion.com/revistas/coleccion/, ISSN 2255-453X

(C) Global Knowledge Academics. Juan Manuel Álvarez

Todos los Derechos Reservados. Permisos: soporte@gkacademics.com 


\section{Introducción}

$\mathrm{E}$

XISTEN MUCHOS TIPOS de orientación, (educativa, profesional, etc.) el presente proyecto se basa en el estudio de la orientación vocacional; desde una nueva perspectiva, que se relaciona con el uso de las tecnologías y medios de comunicación para innovar con una visión y voz, e intentar disminuir la brecha generacional, por ello, este proyecto plantea dos elementos novedosos en la orientación vocacional: el uso de la nuevas tecnologías y la orientación entre pares, (de joven a joven). Puesto que el ser humano vive en un "...mundo lleno de información, que le distrae pero que poco a poco lo convierte en un hombre superficial indiferente, permisivo, en el que anida un gran vacío moral..." (Enrique Rojas; 1992: 14) que hace más compleja la formación de las personas y principalmente, de los estudiantes.

El proyecto de orientación vocacional será creado en función del uso de las nuevas tecnologías de los medios de comunicación (vía Internet); pretende brindar apoyo a todas aquellas personas que quieran ingresar a una formación superior en la Universidad de Guanajuato. La página web intentará orientar al usuario para la efica elección del área profesional a la que pretende unirse, de tal manera que la selección tome en cuenta sus habilidades, intereses, conocimientos y las posibilidades que el entorno le ofrece.

"México y otros países en vías de desarrollo confrontan actualmente un cambio en la forma de proveer educación básica y superior a su población. Cambio que es producto de la acelerada transformación tecnológica..." (Fernando Montero; 1991: 91), por lo cual nuestro país ofrece una educación a distancia desde 1974. Fernando Montero cita a Vanscoit, quién menciona que "...una modalidad extraescolar, que brinda alternativas, a quienes se proponen emprender o continuar su formación, de manera individual y autodidacta, sin la presencia continua de un educador, restricciones de edad, opinión o tiempo..." (Fernando Montero; 1991: 93), se ha convertido en una opción viable para un país cuya mayor población está constituida por jóvenes en proceso de formación, con un gran retraso educativo. En el mundo contemporáneo, el exceso de información se ha convertido en un factor desorientador, además las formas de comunicación se han revolucionado por la intervención de las tecnologías, lo cual ha provocado nuevas problemáticas para los jóvenes y sus procesos formativos.

El objetivo de este proyecto es difundir entre los estudiantes del nivel medio superior, las diferentes carreras que ofrece la Universidad de Guanajuato, considerando las miradas y perspectivas de los propios estudiantes que se forman en dichos programas de estudio, ya que consideramos que la diferencia de edades y la brecha generacional constituyen un obstáculo para la clara y efectiva comunicación.

Se llevará a cabo la creación de una plataforma en internet, con el fi de apoyar a todas aquellas personas que se encuentran con dudas acerca de su formación educativa profesional, creando un proceso como guía de elección de una ocupación para prepararse, ingresar y progresar en ella. La cual se trabajará con la teoría ya existente sobre el tema psicopedagogía y orientación.

\section{Desarrollo}

El mundo social y familiar exige a los jóvenes, un mayor y mejor desempeño profesional y la vida de confort les pide una buena remuneración. Sin embargo, no hemos previsto los mecanismos de autoconocimiento y exploración de las necesidades y gustos formativos de nuestros jóvenes. La orientación vocacional generalmente tiene un perfi de la generación 
mayor hacia la menor, tratándose de un proceso de generaciones de reemplazo; sin embargo, el cambio de los entornos y contextos sociales plantea nuevos horizontes y técnicas de orientación y formación.

Cada vez con mayor preocupación observamos en nuestro entorno, jóvenes confundidos y desorientados por la gran variedad de carreras que se ofertan, y que provocan un estado permanente de estrés y angustia. Existen alumnos que no logran identifica lo que quieren estudiar después de egresar de una educación media superior, ya que hay una gran diversidad de carreras; por consiguiente no tienen claridad sobre lo que pretenden ejercer en el futuro.

\section{a) Antecedentes}

Hablar de orientación es un tema complejo, porque no existe una única variable con la cual se pueda abordar, y hay una lucha constante por delimitar su terminología. En Estados Unidos se comenzó a utilizar el significad de orientación vocacional en su traducción vocacional guidance y actualmente se le conoce como career guidance. Por otra parte, la psicopedagogía se origina a finale del siglo XIX (1882), en los Congresos Higiénico Escolar Pedagógico, y el término orientación vocacional fue utilizado por vez primera en un informe de Frank Pearson en el periodo de 1908. En 1937 National Vocational Guidance Association (NVGA), abordó la definició concreta de la orientación vocacional como “...el proceso por el que se ayuda a alguna persona a elegir una ocupación, a prepararse para ella y progresar en ella..." (Rafael Bisquerra; 1990:1).

En México la orientación vocacional tuvo “...mayor impacto en el primer congreso de los niños que se celebró en 1921" (Barbará Kepowickz; 2004: 224), existió gran evidencia en la falta de un tutor, que orientara a todas aquellas personas que no sabían qué era lo más adecuado para estudiar partir de sus gustos, intereses y habilidades. "La creación del Instituto Politécnico Nacional (IPN) en 1938, dio origen a la primera guía de carreras con mayor interés técnico y económico del país" (Bárbara Kepowickz Malinowska; 2004: 227), puesto que esta institución surgió de la necesidad de crear técnicos competentes; aplicó la orientación educativa como una forma de guiar a los jóvenes en su formación profesional y educativa.

"En los años (1940-1970), período en el que se consolida el modo económico de desarrollo estabilizador, se incrementa el interés por la orientación vocacional" (Barbará Kepowickz Malinowska; 2004:227), y a partir de los 50's la Secretaría de Educación Pública (SEP) comienza a ofrecer los servicios de psicopedagogía, para favorecer la formación profesional requeridos por el país. En 1960 la SEP publica el primer escrito (La orientación educativa y vocacional en la segunda enseñanza), en 1981 las instituciones de educación comprenden la importancia del tema; la SEP creó especialidades para cubrir la necesidad de orientar vocacionalmente a los alumnos, e implementó carreras como psicología educativa, orientación vocacional, escuela normal superior y psicología, pero aún así no tuvo los avances esperados, los alumnos no mostraron interés en estas carreras.

\section{b) Propuestas diversas}

La variedad de las propuestas de orientación lleva a la modernidad y al cambio, pero éste requiere de muchos elementos y tecnologías nuevas y complejas, que cambian de manera continua, demandando la actualización y ajustes, intentado cubrir las necesidades de las 
personas así como su formación. En España se ofrece una orientación por vía internet, creada por Antonio Gutiérrez Gay en 1998.

El enfrentarse a un mundo cambiante complica cada vez más la decisión en la persona al elegir una profesión, con el paso del tiempo se van creando nuevas carreras y como efecto se extiende la variedad, pero al mismo tiempo la complejidad para elegir, por lo cual los sujetos se encuentran en una constante búsqueda para guiarse en su proceso formativo, educativo, psicopedagógico, personal, escolar y otros.

Guadalupe Escamilla Gil (Guadalupe Escamilla Gil; 2004: 21) explica que, el México de nuestros días está enmarcado en un mundo en constante innovación que impacta las formas de vida y las relaciones sociales, todas las maneras en que se intercambia el conocimiento y cómo se resuelve la formación de las nuevas generaciones.

Para Ángel Hernando Gómez (Ángel Hernando Gómez; 2004: 221), la utilización de las Tecnologías de la Información y la Comunicación (TIC's) y la construcción de herramientas de orientación enriquecidas tecnológicamente pueden optimizar y mejorar los procesos de intervención mediante programas. Estas nuevas tecnologías son, sin duda, un complemento y una nueva forma de hacer, que puede y debe aplicarse a todos los modelos de orientación.

Bárbara Kepowicks Malinowska (Bárbara Kepowicks Malinowska; 2004:232), plantea que la revisión de las tendencias a nivel internacional, indica que la orientación se considera para el sistema educativo como un elemento esencial para favorecer la calidad y mejora de la enseñanza.

El mundo está inmerso en las innovaciones para facilitar la forma de vida y el desenvolvimiento de la misma, con ayuda de la tecnología para abrir la puerta del conocimiento y de la formación personal, por lo cual es necesario implementar el uso de las TIC's. Crear buenas herramientas para el mejoramiento de la educación y establecer una forma de ayuda en la cual se les facilite trabajar a los jóvenes, de una manera dinámica, simple y divertida, apoyando en gran medida el conocimiento para ofrecer orientación vocacional, a todas aquellas personas que requieren de una guía para elegir su carrera. Este implemento es muy necesario, ya que existe un gran número de jóvenes que egresan de la educación media superior y se debaten ante la pregunta: “¿Qué me gustaría estudiar?”.

La orientación vocacional es considerada esencial, para mejorar la enseñanza y el desenvolvimiento de los jóvenes en su profesión, y es necesario tener claro lo que se quiere hacer y qué es mejor hacer. Para guiar la formación de la persona el mejoramiento tanto a nivel personal, como también a nivel social.

\section{Conclusión}

La orientación es un implemento necesario para la educación de las personas, pero es muy difícil de abordar, por lo cual se trabajará con especialistas como psicólogos, pedagogos, orientadores vocacionales y licenciados en educación. Este proyecto será trabajado en una plataforma en Internet por lo cual se requiere de ingenieros en sistemas computacionales y licenciados en diseño gráfico esto es de gran utilidad porque en México no existe una herramienta orientativa-educativa-vocacional, en un sistema tecnológico como el espacio web; éste nos permitirá no sólo reflexiona sobre la situación actual que guarda la orientación vocacional, sino también innovar y proponer con base en los especialistas; modificacione y actualizaciones pertinentes a los instrumentos y procesos clásicos de la orientación vocacional, más acordes con la realidad que vivimos los jóvenes. 


\section{Referencias}

Bisquerra, Rafael (1990). Orientación psicopedagógica para la prevención y el desarrollo. Barcelona, Ed. Boixareu Universitaria.

Escamilla Gil, Guadalupe (2004). Las tendencias de la matricula de educación superior en México. México, Ed. Revista Mexicana de Orientación Educativa.

Hernando Gómez, Ángel (2009). Una WebQuest para la orientación vocacional y profesional en Bachillerato. Ed. Revista Redalyc.

Kepowicks, Barbara (2004). Educación, Estado y Sociedad. (IIEDUG), Ed. Instituto de Investigación en Educación de la Universidad de Guanajuato.

Montera, Fernando (1999). Educación a distancia orden y caos: aspectos de la postmodernidad. México, Ed. Taller abierto.

Rojas, Enrique (1992). El hombre light. México, Ed. Planeta mexicana.

Sierra, Francisco (2000). Introducción a la teoría de la comunicación educativa. España, Ed. MAD.

\section{Sobre el Autor}

Juan Manuel Álvarez: Egresado de un bachillerato técnico con especialidad en informática (sistemas computacionales) con el perfi de físico-matemático. Estudiante de la Licenciatura en Educación de la Universidad de Guanajuato en el Departamento de Educación de la División de Ciencias Sociales y humanidades. En un futuro egresando con un perfi en orientación y gestión educativa. Actualmente, apoyo de profesor de orientación en dos cátedras (Proyecto de vida con los estudiantes de 2do. Grado, y Orientación profesional con los de 4to. Semestre), en el bachillerato oficia de la Universidad de Guanajuato, en la ciudad de Guanajuato. 